astro-ph/9811431

UFIFT-HEP-98-17

\title{
One Loop Back Reaction On Power Law Inflation
}

\author{
L. R. Abramo*† and R. P. Woodard ${ }^{\ddagger}$ \\ Department of Physics \\ University of Florida \\ Gainesville, FL 32611 USA
}

\begin{abstract}
We consider quantum mechanical corrections to a homogeneous, isotropic and spatially flat geometry whose scale factor expands classically as a general power of the co-moving time. The effects of both gravitons and the scalar inflaton are computed at one loop using the manifestly causal formalism of Schwinger [1] with the Feynman rules recently developed by Iliopoulos et al. [2]. We find no significant effect, in marked contrast with the result obtained by Mukhanov et al. [3, 4] for chaotic inflation based on a quadratic potential. By applying the canonical technique of Mukhanov et al. to the exponential potentials of power law inflation, we show that the two methods produce the same results, within the approximations employed, for these backgrounds. We therefore conclude that the shape of the inflaton potential can have an enormous impact on the one loop back-reaction.
\end{abstract}

PACS numbers: 04.60.-m, 98.80.Cq

* Address after May 1, 1999: Theoretische Physik, Ludwig Maximilians Universität, Theresienstr. 37, D-80333 MÜNCHEN, GERMANY

$\dagger$ e-mail: abramo@phys.ufl.edu

$\ddagger$ e-mail: woodard@phys.ufl.edu 


\section{Introduction}

In recent papers, Mukhanov, Abramo and Brandenberger [3], 1] have investigated the problem of the back reaction of quantum fluctuations in chaotic inflation. Using canonical quantization, they calculated the one loop effective energy-momentum tensor induced by quantum fluctuations of the matter and metric fields. They found that the expansion rate of the background Friedmann-Robertson-Walker space-time slows down as a result of quantum deformations to the equations of motions.

Is this result generic for all inflationary models? In other words, does back reaction slow down the expansion rate $H$ in scenarios other than chaotic inflation? In this paper we extend the work of Mukhanov et al. to a very different class of inflationary models, the so-called power-law inflation [5] in which the expansion of the universe is given by a scale factor $a(t) \propto t^{s}$ with $s>1$. For that purpose we employ the standard formalism of covariant quantization, using the Feynman rules for these backgrounds that have been worked out by Iliopoulos, Tomaras, Tsamis and Woodard [2].

We find that the one loop back reaction in power-law models is negligible and stays negligible through the inflation of the universe. By comparison, in the chaotic models the one loop infrared corrections can become nonperturbatively large at late times.

The effect discussed by Mukhanov et al. is closely related to the one found in earlier work by Tsamis and Woodard [6] in the context of pure gravity with a cosmological constant. Both mechanisms have a simple physical origin: the enhancement of zero-point energy of the quantum fields by the expansion of the universe, also known in the literature as superadiabatic amplification. Virtual quanta of cosmological wavelengths become trapped by the expansion of the universe and are unable to recombine. Gravitational interactions between these virtual pairs, being always attractive, slow the expansion rate. Because gravitational interactions are very weak, back reaction takes a long time before it can become a sizeable effect. As an infrared effect that derives from quantum fluctuations whose wavelengths are comparable

to the Hubble radius $H^{-1}$, back reaction can be studied in the context of general relativity, whatever is the ultimate theory of gravity円.

\footnotetext{
${ }^{1}$ This is precisely the reason why cosmologists are able to predict the seeds of structure formation from quantum fluctuations in inflationary models without regard to the true theory of quantum gravity[9.
} 
A second point of this paper is to prove that results obtained by covariant quantization should coincide with those worked out using canonical quantization. This is relevant to the validity and interpretation of the canonical calculation, which was used by Mukhanov et al. to derive their results and has recently been challenged by Unruh [0]. In a separate paper [8] we go further and show that covariant quantization yields the same physical results as canonical quantization in the case of the chaotic inflation models as well.

The present paper is organized as follows. In section 2 we describe the perturbative background. We write the Feynman rules for the gravity-scalar system (taken from Iliopoulos et al [2]) in section 3. In section 4 we show how to use Schwinger's formalism to obtain expectation values for the metric and matter fields from the amputated 1-point functions of the theory. In section 5 we derive the infrared parts of coincident propagators. Our results are given in section 6 . In section 7 we re-derive those results using gauge-fixed canonical quantization, and show that the two methods give identical results, namely that one loop back reaction is negligible in power-law inflation. Section 8 discusses the implications of this work.

\section{Classical background}

The system we consider is general relativity with a minimally coupled scalar field:

$$
\mathcal{L}=\frac{1}{16 \pi G} R \sqrt{-g}-\frac{1}{2} \partial_{\mu} \varphi \partial_{\nu} \varphi g^{\mu \nu} \sqrt{-g}-V(\varphi) \sqrt{-g} .
$$

The background is comprised of a flat, homogeneous and isotropic metric

$$
d s_{0}^{2}=-d t^{2}+a_{0}^{2}(t) d \vec{x} \cdot d \vec{x}=\Omega^{2}(\eta)\left(-d \eta^{2}+d \vec{x} \cdot d \vec{x}\right)
$$

and scalar field $\varphi_{0}(t)$. The conformal time $\eta$ is related to comoving time by $d t=\Omega(\eta) d \eta$. In this paper we examine those scalar potentials which drive

power-law expansions, that is, the scale factor grows as a function of time according to

$$
a_{0}(t)=\left(1+\frac{H_{\mathrm{i}} t}{s}\right)^{s},
$$

where $s$ and $H_{\mathrm{i}}$ are constants. In terms of conformal time, 


$$
\Omega(\eta)=\left(\frac{\eta_{i}}{\eta}\right)^{\frac{s}{s-1}},
$$

and $\eta_{i}=-\frac{s-1}{s} H_{\mathrm{i}}^{-1}$. The advantage of this class of expansion laws is that the Feynman rules are already known[2].

We now write some useful formulas for the background quantities. The expansion rate is given by the logarithmic derivative of the scale factor with respect to comoving time,

$$
H \equiv \frac{\dot{a}_{0}}{a_{0}}=\frac{\Omega^{\prime}}{\Omega^{2}}=H_{\mathrm{i}} \Omega^{-\frac{1}{s}},
$$

where a dot indicates $\partial / \partial t$ and a prime denotes $\partial / \partial \eta=\Omega \partial / \partial t$.

The Einstein field equations are

$$
\begin{aligned}
3 H^{2} & =\frac{1}{2} \kappa^{2}\left[\frac{1}{2} \dot{\varphi}_{0}^{2}+V\left(\varphi_{0}\right)\right], \\
-2 \dot{H}-3 H^{2} & =\frac{1}{2} \kappa^{2}\left[\frac{1}{2} \dot{\varphi}_{0}^{2}-V\left(\varphi_{0}\right)\right],
\end{aligned}
$$

and the integrability condition for this system is given by the equation of motion for the background scalar field,

$$
\ddot{\varphi}_{0}+3 H \dot{\varphi}_{0}+V_{, \varphi}\left(\varphi_{0}\right)=0 .
$$

In the expressions above, $\kappa^{2}=16 \pi G$ is the loop-counting parameter of perturbative quantum gravity, $V(\varphi)$ is one of the scalar field potentials of powerlaw inflation models and $V_{, \varphi} \equiv \partial V / \partial \varphi$.

It is useful to invert the Einstein equations and write the scalar field quantities in terms of the Hubble parameter $H$ and its derivative $\dot{H}$ :

$$
\begin{aligned}
\dot{\varphi}_{0}^{2} & =-\frac{4}{\kappa^{2}} \dot{H}, \\
V\left(\varphi_{0}\right) & =\frac{2}{\kappa^{2}}\left(\dot{H}+3 H^{2}\right) .
\end{aligned}
$$

In terms of the conformal scale factor, the expressions are 


$$
\begin{aligned}
\varphi_{0}^{\prime 2} & =\frac{4}{\kappa^{2}}\left[-\frac{\Omega^{\prime \prime}}{\Omega}+2\left(\frac{\Omega^{\prime}}{\Omega}\right)^{2}\right], \\
V\left(\varphi_{0}\right) & =\frac{2}{\kappa^{2}} \frac{1}{\Omega^{2}}\left[\frac{\Omega^{\prime \prime}}{\Omega}+\left(\frac{\Omega^{\prime}}{\Omega}\right)^{2}\right] .
\end{aligned}
$$

If we now substitute the expansion law (4) into expressions (11) and (12), it is easy to obtain the scalar field potentials which correspond to power-law inflation [5]:

$$
\begin{aligned}
\varphi_{0} & =\frac{2}{\sqrt{s}} \frac{1}{\kappa} \ln (\Omega) \\
V(\varphi) & =6\left(1-\frac{1}{3 s}\right) \frac{H_{\mathrm{i}}^{2}}{\kappa^{2}} \exp \left[-\frac{\kappa}{\sqrt{s}} \varphi\right] .
\end{aligned}
$$

The parameter $s$ therefore regulates the steepness of the scalar potential as well as the rate of expansion. Notice that the equation of state for the energy density and pressure of the scalar field is

$$
w=\frac{p}{\rho}=\frac{3 H^{2}}{-2 \dot{H}-3 H^{2}}=-1+\frac{2}{3 s} .
$$

In the limit $s \rightarrow \infty$ we recover exponential inflation and the equation of state for de Sitter space, $w=-1$.

\section{Quantum theory: Feynman rules}

Before laying down the quantum theory, we would like to address an objection that is too often raised: that general relativity is not a perturbatively consistent quantum theory of gravity, and so cannot be used to study quantum effects.

That judgment is only partially correct. The back reaction of quantum fluctuations described here is an infrared effect, caused by fluctuations with cosmological wavelengths, and we know, for example from the Fermi theory of neutrinos 10], that infrared physics can be studied by the low-energy effective theory, regardless of the renormalizability of that theory. Conversely, 
whatever the ultraviolet behavior of the true quantum theory of gravity may turn out to be, it will not be affected by these infrared effects. As long as we are careful not to introduce a spurious time dependence through the ultraviolet regularization, back reaction is given at late times by ultraviolet finite terms whose form is entirely controlled by the low-energy theory.

We also note that quantizing some of the gravitational degrees of freedom is crucial to all derivations of the density perturbations and cosmic microwave background anisotropies in inflationary cosmology [9]. Both we and Mukhanov et al. just carry the calculations out to the next stage, and ask what effects quantum fluctuations might have on the background in which they propagate.

The goal of this section is to summarize the Feynman rules for the Lagrangian (1). The fundamental fields are the metric and the scalar field,

$$
\begin{aligned}
g_{\mu \nu} & =\Omega^{2}\left(\eta_{\mu \nu}+\kappa \psi_{\mu \nu}\right) \equiv \Omega^{2} \tilde{g}_{\mu \nu} \\
\varphi & =\varphi_{0}+\phi .
\end{aligned}
$$

Here $\psi_{\mu \nu}$ is the pseudo-graviton field, whose indices are raised and lowered with the Lorentz metric $\eta_{\mu \nu}$.

Both the scalar-scalar and the graviton-scalar interaction parts of the Lagrangian can be easily expanded in perturbation theory from the fundamental Lagrangian (II). The pure gravitational interactions are more complicated, however, after some integrations by parts the whole Lagrangian reduces to the simple expression,

$$
\begin{aligned}
\mathcal{L}_{\mathrm{inv}}= & \Omega^{2} \sqrt{-\widetilde{g}} \tilde{g}^{\alpha \beta} \widetilde{g}^{\rho \sigma} \widetilde{g}^{\mu \nu}\left[\frac{1}{2} \psi_{\alpha \rho, \mu} \psi^{\nu \sigma, \beta}-\frac{1}{2} \psi_{\alpha \beta, \rho} \psi^{\sigma \mu, \nu}+\frac{1}{4} \psi_{\alpha \beta, \rho} \psi^{\mu \nu, \sigma}\right. \\
& \left.-\frac{1}{4} \psi_{\alpha \rho, \mu} \psi^{\beta \sigma, \nu}\right]+\Omega \Omega^{\prime} \sqrt{-\widetilde{g}} \widetilde{g}^{\rho \sigma} \widetilde{g}^{\mu \nu} \psi_{\rho \sigma, \mu} \psi_{0 \nu}-\Omega^{2} \sqrt{-\widetilde{g}} \varphi_{0}^{\prime} \phi_{, \mu} \widetilde{g}^{0 \mu} \\
& -\frac{1}{2} \Omega^{2} \sqrt{-\widetilde{g}} \phi_{, \mu} \phi_{, \nu} \widetilde{g}^{\mu \nu}-\Omega^{4} \sqrt{-\widetilde{g}} \sum_{n=1}^{\infty} \frac{1}{n !} \frac{\partial^{n} V\left(\varphi_{0}\right)}{\partial \varphi^{n}} \phi^{n}
\end{aligned}
$$

up to total derivative terms.

Gauge fixing is achieved by adding a gauge fixing term and the corresponding ghost action to the invariant Lagrangian: 


$$
\mathcal{L}_{\mathrm{BRS}}=\mathcal{L}_{\mathrm{inv}}-\frac{1}{2} \eta^{\mu \nu} F_{\mu} F_{\nu}-\Omega \bar{\omega}^{\mu} \delta F_{\mu}
$$

The symbol $\delta F_{\mu}$ represents the variation of the gauge fixing functional under an infinitesimal diffeomorphism parameterized by the ghost field $\omega^{\mu}$. We follow Iliopoulos et al. in the choice of the gauge fixing functional:

$$
F_{\mu}=\Omega\left(\psi_{\mu, \nu}^{\nu}-\frac{1}{2} \psi_{, \mu}-2 \frac{\Omega^{\prime}}{\Omega} \psi_{\mu 0}+\eta_{\mu 0} \kappa \varphi_{0}^{\prime} \phi\right) .
$$

A great advantage of this gauge is that it decouples the tensor structure of the propagators from their dependence on spacetime.

The quadratic part of the gauge-fixed Lagrangian (19) can be written, up to ghost contributions, in terms of a kinetic operator in "super-matrix" representation:

$$
\mathcal{D} \equiv\left(\begin{array}{ll}
D_{\mu \nu}^{\rho \sigma} & -\frac{2 \sqrt{s}}{s-1} \frac{\Omega^{2}}{\eta^{2}} t^{\rho} t^{\sigma} \\
-\frac{2 \sqrt{s}}{s-1} \frac{\Omega^{2}}{\eta^{2}} t_{\mu} t_{\nu} & \Omega\left[\partial^{2}+\frac{2 s^{2}-3 s+2}{(s-1)^{2}} \frac{1}{\eta^{2}}\right] \Omega
\end{array}\right),
$$

where $t_{\mu}=\eta_{\mu 0}$. To get back the quadratic terms of the Lagrangian (19), just multiply the super-matrix $\mathcal{D}$ on the left by $\left(\psi^{\mu \nu} \phi\right)$, and on the right by its transpose. The kinetic operator $D_{\mu \nu}^{\rho \sigma}$ is given by

$$
\begin{aligned}
D_{\mu \nu}{ }^{\rho \sigma} \equiv & {\left[\frac{1}{2} \bar{\delta}_{\mu}^{(\rho} \bar{\delta}_{\nu}^{\sigma)}-\frac{1}{4} \eta_{\mu \nu} \eta^{\rho \sigma}-\frac{1}{2} t_{\mu} t_{\nu} t^{\rho} t^{\sigma}\right] D_{A} } \\
& +\left[t_{\mu} t_{\nu} t^{\rho} t^{\sigma}-t_{(\mu} \bar{\delta}_{\nu)}^{(\rho} t^{\sigma)}\right] D_{B},
\end{aligned}
$$

where barred tensor symbols denotes that the zero components have been projected out: $\bar{\delta}_{\mu}^{\nu} \equiv \delta_{\mu}^{\nu}+t_{\mu} t^{\nu}$. The kinetic operators above are given by

$$
\begin{aligned}
D_{A}^{s} & \equiv \Omega\left[\partial^{2}+\frac{2 s^{2}-s}{(1-s)^{2}} \frac{1}{\eta^{2}}\right] \Omega, \\
D_{B}^{s} & \equiv \Omega\left[\partial^{2}+\frac{s}{(1-s)^{2}} \frac{1}{\eta^{2}}\right] \Omega .
\end{aligned}
$$

The off-diagonal term in (21), coupling $\psi_{00}$ to $\phi$, can be removed by a change of variables [2]: 


$$
\begin{aligned}
\zeta_{i j} & \equiv \psi_{i j}-\delta_{i j} \psi_{00}, \\
\chi & \equiv \phi \sin \theta+\psi_{00} \cos \theta, \\
v & \equiv \phi \cos \theta-\psi_{00} \sin \theta,
\end{aligned}
$$

where $\tan ^{2} \theta=s$ and Latin letters denote spatial indices.

In terms of the new fields (25)-(27) the quadratic, gauge-fixed Lagrangian is:

$$
\begin{aligned}
\mathcal{L}_{B R S}^{(2)}= & \frac{1}{2} \zeta_{i j} D_{A}^{s} \zeta_{r s}\left[\frac{1}{2} \delta_{i(r} \delta_{s) j}-\frac{1}{4} \delta_{i j} \delta_{r s}\right]+\frac{1}{2} \zeta_{0 i} D_{B}^{s} \zeta_{0 j}\left[-\delta_{i j}\right] \\
& +\frac{1}{2} v D_{C}^{s} v+\frac{1}{2} \chi D_{A}^{s} \chi+\bar{\omega}^{i} D_{A}^{s} \omega_{i}+\bar{\omega}^{0} D_{B}^{s} \omega_{0},
\end{aligned}
$$

where $D_{A}^{s}$ and $D_{B}^{s}$ were defined above in (23)-(24), and $D_{C}^{s}$ is given by

$$
D_{C}^{s} \equiv \Omega\left[\partial^{2}+\frac{2-s}{(1-s)^{2}} \frac{1}{\eta^{2}}\right] \Omega .
$$

The quantization of this system is trivial. There are three modes associated with the three kinetic operators:

$$
D_{I}^{s} \Psi(\eta, k, I)=0 .
$$

We note for now that by Eq. (30) and the definitions of the kinetic operators (23)-(24) and (29), the mode functions are proportional to Hankel functions $H_{\nu_{I}}^{(1,2)}(k \eta)$ where the labels $\nu_{I}(s)$ depends on the parameter $s$. In the limit where $k \eta \rightarrow \infty$ we find that the modes proportional to $H_{\nu_{I}}^{(2)}$ have negative frequency, while those proportional to $H_{\nu_{I}}^{(1)}=H_{\nu_{I}}^{(2) *}$ have positive frequency.

The fundamental fields $\psi$ and $\phi$ can be expressed in terms of the mode functions above. The scalar field, for example, is decomposed in the following manner:

$$
\begin{aligned}
\phi(\eta, \vec{x}) & =\sum_{I} f_{I} \psi_{I}(\eta, \vec{x}) \\
& =\sum_{I} f_{I} \int \frac{d^{3} k}{(2 \pi)^{3}}\left[e^{i \vec{k} \cdot \vec{x}} \Psi(\eta, k, I) a(\vec{k}, I)+e^{-i \vec{k} \cdot \vec{x}} \Psi^{*}(\eta, k, I) a^{\dagger}(\vec{k}, I)\right] .
\end{aligned}
$$


The weights $f_{I}$ can be found by inverting relations (26)-(27), so in the example above their values are $f_{A}=\sin \theta, f_{C}=\cos \theta$ and $f_{B}=0$.

The quanta of negative energy are associated with the negative frequency modes $\Psi$ and the annihilation operators $a(\vec{k}, I)$. Therefore the vacuum of the theory $|0\rangle$ is defined as the state that is annihilated by all $a(\vec{k}, I)$ 's and $I=A, B, C$.

The creation and annihilation operators $a^{\dagger}$ and $a$ obey the usual commutation relations

$$
\left[a(\vec{k}, I), a^{\dagger}\left(\vec{k}^{\prime}, I^{\prime}\right)\right]=\delta_{I I^{\prime}} \delta^{(3)}\left(\vec{k}-\vec{k}^{\prime}\right),
$$

and the mode functions are normalized by

$$
\Psi(\eta, k, I) \Psi^{\prime *}(\eta, k, I)-\Psi^{*}(\eta, k, I) \Psi^{\prime}(\eta, k, I)=i \Omega^{-2} .
$$

The scalar field propagator is defined by

$$
i \Delta_{\phi}\left(x ; x^{\prime}\right) \equiv\left\langle 0\left|T\left\{\phi(x) \phi\left(x^{\prime}\right)\right\}\right| 0\right\rangle .
$$

We shall need pure graviton and mixed propagators as well. All these can be expressed in terms of propagators of the diagonal fields $\left\langle 0\left|T\left\{\psi_{I}(x) \psi_{I}\left(x^{\prime}\right)\right\}\right| 0\right\rangle$, which can be conveniently written in momentum space as

$$
\begin{aligned}
i \Delta_{I}\left(x ; x^{\prime}\right)=\int \frac{d^{3} k}{(2 \pi)^{3}} e^{-\varepsilon k} e^{i \vec{k} \cdot\left(\vec{x}-\vec{x}^{\prime}\right)} \times & {\left[\theta\left(\eta-\eta^{\prime}\right) \Psi(\eta, k, I) \Psi^{*}\left(\eta^{\prime}, k, I\right)\right.} \\
+ & \left.\theta\left(\eta^{\prime}-\eta\right) \Psi^{*}(\eta, k, I) \Psi\left(\eta^{\prime}, k, I\right)\right]
\end{aligned}
$$

with $I=A, B, C$. Explicit formulae for these propagators in the infrared limit can be found in section 5 .

Finally, we transform from the diagonal variables $\zeta_{i j}, v$ and $\chi$ back to the original pseudo-graviton $\psi_{\mu \nu}$ and scalar field $\phi$ by using relations (25)-(27). The set of propagators below is the main result of this section:

$$
\begin{aligned}
\left\langle 0\left|T\left\{\psi_{i j}(x) \psi_{r s}\left(x^{\prime}\right)\right\}\right| 0\right\rangle= & i \Delta_{A}^{s}\left(x ; x^{\prime}\right) 2\left[\delta_{i(r} \delta_{s) j}-\delta_{i j} \delta_{r s}\right] \\
& +i\left[\Delta_{A}\left(x ; x^{\prime}\right)+s \Delta_{C}^{s}\left(x ; x^{\prime}\right)\right] \frac{1}{1+s} \delta_{i j} \delta_{r s} \\
\left\langle 0\left|T\left\{\psi_{0 i}(x) \psi_{0 r}\left(x^{\prime}\right)\right\}\right| 0\right\rangle= & -i \Delta_{B}^{s}\left(x ; x^{\prime}\right) \delta_{i r}
\end{aligned}
$$




$$
\begin{aligned}
\left\langle 0\left|T\left\{\psi_{00}(x) \psi_{00}\left(x^{\prime}\right)\right\}\right| 0\right\rangle & =i \Delta_{A}^{s}\left(x ; x^{\prime}\right) \frac{1}{1+s}+i \Delta_{C}^{s}\left(x ; x^{\prime}\right) \frac{s}{1+s} \\
\left\langle 0\left|T\left\{\psi_{00}(x) \psi_{i j}\left(x^{\prime}\right)\right\}\right| 0\right\rangle & =\left\langle 0\left|T\left\{\psi_{00}(x) \psi_{00}\left(x^{\prime}\right)\right\}\right| 0\right\rangle \delta_{i j} \\
\left\langle 0\left|T\left\{\psi_{00}(x) \phi\left(x^{\prime}\right)\right\}\right| 0\right\rangle & =-i \Delta_{A}^{s}\left(x ; x^{\prime}\right) \frac{\sqrt{s}}{1+s}+i \Delta_{C}^{s}\left(x ; x^{\prime}\right) \frac{\sqrt{s}}{1+s} \\
\left\langle 0\left|T\left\{\psi_{i j}(x) \phi\left(x^{\prime}\right)\right\}\right| 0\right\rangle & =\left\langle 0\left|T\left\{\psi_{00}(x) \phi\left(x^{\prime}\right)\right\}\right| 0\right\rangle \delta_{i j} \\
\left\langle 0\left|T\left\{\phi(x) \phi\left(x^{\prime}\right)\right\}\right| 0\right\rangle & =i \Delta_{A}^{s}\left(x ; x^{\prime}\right) \frac{s}{1+s}+i \Delta_{C}^{s}\left(x ; x^{\prime}\right) \frac{1}{1+s} \\
\left\langle 0\left|T\left\{\bar{\omega}_{0}(x) \bar{\omega}_{0}\left(x^{\prime}\right)\right\}\right| 0\right\rangle & =-i \Delta_{B}^{s}\left(x ; x^{\prime}\right) \\
\left\langle 0\left|T\left\{\bar{\omega}_{i}(x) \bar{\omega}_{j}\left(x^{\prime}\right)\right\}\right| 0\right\rangle & =i \Delta_{A}^{s}\left(x ; x^{\prime}\right) \delta_{i j} .
\end{aligned}
$$

\section{Attaching external lines}

Back reaction can change the dynamics of the homogeneous and isotropic background in three ways: through a redefinition of the time slicing (corrections to $\left\langle\psi_{00}\right\rangle$ ), through a change in the scale factor (corrections to the trace $\left.\left\langle\psi_{i i}\right\rangle\right)$ and through a shift in the scalar field that drives inflation. Because the initial state is homogeneous and isotropic, the expectation values of the pseudo-graviton and scalar are functions only of time:

$$
\begin{aligned}
\left\langle 0\left|\kappa \psi_{\mu \nu}(\eta, \vec{x})\right| 0\right\rangle & =A(\eta) \bar{\eta}_{\mu \nu}+C(\eta) t_{\mu} t_{\nu} \\
\langle 0|\kappa \phi(\eta, \vec{x})| 0\rangle & =D(\eta) .
\end{aligned}
$$

The expectation values of the full metric and scalar field on the state $|0\rangle$ are then

$$
\begin{aligned}
\left\langle 0\left|d s^{2}\right| 0\right\rangle & =\Omega^{2}\left[-(1-C) d \eta^{2}+(1+A) d \vec{x} \cdot d \vec{x}\right] \\
& =-d t^{2}+a^{2}(t) d \vec{x} \cdot d \vec{x}, \\
\langle 0|\kappa \varphi| 0\rangle & =\frac{2}{\sqrt{s}} \ln (\Omega)+D,
\end{aligned}
$$

where $t(\eta)=\int_{\eta_{i}}^{\eta} \Omega\left(\eta^{\prime}\right) d \eta^{\prime}$ is the background comoving time and the scale factor in comoving time is given by 


$$
a^{2}(t)=a_{0}^{2}(t)\left\{1+A[\eta(t)]+H(t) \int_{0}^{t} d t^{\prime} C\left[\eta\left(t^{\prime}\right)\right]\right\}
$$

We don't measure expectation values directly, but rather combinations of them that constitute physical observables. The observables should be independent of gauge-fixing[1]. In the case of homogeneous and isotropic backgrounds, one such physical observable is the effective expansion rate $H_{\text {eff }}$ defined as the logarithmic derivative of the scale factor with respect to comoving time:

$$
\begin{aligned}
H_{\mathrm{eff}} & \equiv \frac{d \ln a(t)}{d t} \\
& =H(t)\left[1+\frac{1}{2} C(t)+\frac{\dot{A}(t)}{2 H}+\frac{\dot{H}}{2 H} \int_{0}^{t} d t^{\prime} C\left(t^{\prime}\right)+\ldots\right] .
\end{aligned}
$$

Rather than computing the 1-point functions $A, C$ and $D$ directly, we will compute the amputated 1-point functions instead, and then attach the external lines to find the 1-point functions. The amputated 1-point functions are defined as

$$
\begin{gathered}
\left(\begin{array}{ll}
D_{\mu \nu}^{\rho \sigma} & -\frac{2 \sqrt{s}}{s-\frac{\Omega^{2}}{\eta^{2}}} t^{\rho} t^{\sigma} \\
-\frac{2 \sqrt{s}}{s-1} \frac{\Omega^{2}}{\eta^{2}} t_{\mu} t_{\nu} & \Omega\left[\partial^{2}+\frac{2 s^{2}-3 s+2}{(s-1)^{2}} \frac{1}{\eta^{2}}\right] \Omega
\end{array}\right) \times\left(\begin{array}{c}
\left\langle 0\left|\kappa \psi_{\rho \sigma}\right| 0\right\rangle \\
\langle 0|\kappa \phi| 0\rangle
\end{array}\right) \\
\equiv\left(\begin{array}{c}
\alpha(\eta) \bar{\eta}_{\mu \nu}+\gamma(\eta) t_{\mu} t_{\nu} \\
\delta(\eta)
\end{array}\right)
\end{gathered}
$$

where the matrix on the left is the same differential operator defined above in (21). Substituting the expectation values (37) and (38) gives

$$
\begin{aligned}
\alpha(\eta) & =-\frac{1}{4} D_{A}(A-C) \\
\gamma(\eta) & =-\frac{3}{4} D_{A}(A-C)+D_{B} C-\frac{2 \sqrt{s}}{s-1} \frac{\Omega^{2}}{\eta^{2}} D \\
\delta(\eta) & =\Omega\left[-\frac{d^{2}}{d \eta^{2}}+\frac{2 s^{2}-3 s+2}{(s-2)^{2}} \frac{1}{\eta^{2}}\right] \Omega D-\frac{2 \sqrt{s}}{s-1} \frac{\Omega^{2}}{\eta^{2}} C
\end{aligned}
$$


Since we are calculating expectation values, we must employ Schwinger's formalism [1] instead of the usual rules for "in-out" matrix elements. For the one-loop 1-point functions, the only difference is that in Schwinger's formalism the external lines are retarded propagators. In order to enforce this choice of external propagator we fix retarded boundary conditions such that the 1-point functions $A(\eta), C(\eta)$ and $D(\eta)$, along with their time derivatives, vanish on the initial surface $\eta=\eta_{i}$.

The 1-point functions are obtained by attaching the external legs, i.e., by inverting the coupled differential equations (44)-(46) with appropriate boundary conditions. After a change of variables and some algebra, one obtains

$$
\begin{aligned}
A=\frac{1}{D_{A}} & {\left[-4 \alpha+\frac{1}{s+1}(3 \alpha+\gamma)-\frac{\sqrt{s}}{s+1} \delta\right] } \\
& +\frac{1}{D_{C}}\left[\frac{s}{s+1}(3 \alpha+\gamma)+\frac{\sqrt{s}}{s+1} \delta\right], \\
C=\frac{1}{D_{A}} & {\left[\frac{1}{s+1}(3 \alpha+\gamma)-\frac{\sqrt{s}}{s+1} \delta\right] } \\
& +\frac{1}{D_{C}}\left[\frac{s}{s+1}(3 \alpha+\gamma)+\frac{\sqrt{s}}{s+1} \delta\right], \\
D=\frac{1}{D_{A}} & {\left[-\frac{\sqrt{s}}{s+1}(3 \alpha+\gamma)+\frac{s}{s+1} \delta\right] } \\
& +\frac{1}{D_{C}}\left[\frac{\sqrt{s}}{s+1}(3 \alpha+\gamma)+\frac{1}{s+1} \delta\right],
\end{aligned}
$$

where $D_{A}$ and $D_{C}$ have been defined in (23) and (29). Notice that since $\alpha, \gamma$ and $\delta$ are functions only of time, the non-local operators $1 / D_{I}$ in the expressions above denote two time integrations. The explicit representations for the inverted propagators are

$$
\begin{aligned}
\frac{1}{D_{A}} f(\eta) & =-\int_{\eta_{i}}^{\eta} d \eta^{\prime} \Omega^{-2}\left(\eta^{\prime}\right) \int_{\eta_{i}}^{\eta^{\prime}} d \eta^{\prime \prime} f\left(\eta^{\prime \prime}\right) \\
\frac{1}{D_{C}} f(\eta) & =-\Omega^{-2+\frac{2}{s}}(\eta) \int_{\eta_{i}}^{\eta} d \eta^{\prime} \Omega^{2-\frac{4}{s}}\left(\eta^{\prime}\right) \int_{\eta_{i}}^{\eta^{\prime}} d \eta^{\prime \prime} \Omega^{-2+\frac{2}{s}}\left(\eta^{\prime \prime}\right) f\left(\eta^{\prime \prime}\right)
\end{aligned}
$$


where the lower limits of the integrals make clear that

$$
\begin{gathered}
A\left(\eta_{i}\right)=C\left(\eta_{i}\right)=D\left(\eta_{i}\right)=0, \\
A^{\prime}\left(\eta_{i}\right)=C^{\prime}\left(\eta_{i}\right)=D^{\prime}\left(\eta_{i}\right)=0 .
\end{gathered}
$$

It is useful to derive explicit formulas for the integrals (50)-(51) when the integrand has the same functional form as the amputated 1-point functions. The fastest-growing contributions to the amputated 1-point functions have a time dependence $\propto \Omega^{4-2 / s+\epsilon}$ (see next section), and for such terms the integrals (50)-(51) reduce to

$$
\begin{aligned}
\frac{1}{D_{A}}\left(\Omega^{4-\frac{2}{s}+\epsilon}\right)= & \frac{s}{H_{\mathrm{i}}^{2} \epsilon(3 s-1+s \epsilon)} \\
\times & \left(-\Omega^{\epsilon}+\frac{3 s-1+s \epsilon}{3 s-1}-\frac{s \epsilon}{3 s-1} \Omega^{-\frac{3 s-1}{s}}\right) \\
\frac{1}{D_{C}}\left(\Omega^{4-\frac{2}{s}+\epsilon}\right)= & \frac{s^{2}}{H_{\mathrm{i}}^{2}(s+1+s \epsilon)(2 s-2+s \epsilon)} \\
& \times\left(-\Omega^{\epsilon}+\frac{2 s-2+s \epsilon}{s-3} \Omega^{-\frac{s+1}{s}}-\frac{s+1+s \epsilon}{s-3} \Omega^{-2 \frac{s-1}{s}}\right) .
\end{aligned}
$$

\section{$5 \quad$ Infrared parts of coincident propagators}

In the next section we will sum the diagrams that contribute to the amputated 1-point functions $\alpha, \gamma$ and $\delta$. At one loop, the amputated 1-point functions are found by acting with the 3 -point vertex operators on the propagators $i \Delta_{I}\left(x ; x^{\prime}\right)$ and then taking the coincidence limit $x \rightarrow x^{\prime}$. In this section we study the coincidence limits of the propagators and point out those terms which can contribute to back reaction.

The three diagonal propagators can be expressed in terms of the mode functions $\Psi(\eta, k, I)$ which obey equations (30). After a change of variables

$$
\Psi(\eta, k, I)=(k \eta)^{w} h(k \eta, I) \quad, \quad w=\frac{3 s-1}{2(s-1)},
$$

we can solve Eqs. (30) in terms of Hankel functions $H_{\nu_{I}(s)}^{(1,2)}(k \eta)$ of the first and second kind, where the indices $\nu_{I}(s)$ are given in terms of the parameter $s$ as 21 


$$
\begin{aligned}
& \nu_{A}=\frac{3}{2}+\frac{1}{s-1}, \\
& \nu_{B}=\frac{1}{2}+\frac{1}{s-1}, \\
& \nu_{C}=\frac{1}{2}-\frac{1}{s-1} .
\end{aligned}
$$

The normalization of the mode functions is given by (33). The Hankel functions, on their own account, obey the identities

$$
\begin{aligned}
H_{\nu}^{(1)^{*}}(y) & =H_{\nu}^{(2)}(y), \\
H_{\nu}^{(2)}(y) \frac{d}{d y} H_{\nu}^{(1)}(y)-H_{\nu}^{(1)}(y) \frac{d}{d y} H_{\nu}^{(2)}(y) & =\frac{4 i}{\pi} \frac{1}{y},
\end{aligned}
$$

which imply that the two linearly independent, normalized mode functions are given by

$$
\begin{aligned}
\Psi(\eta, k, I) & =\frac{1}{2} \Omega^{-1} \sqrt{\pi \eta} H_{\nu_{I}}^{(2)}(k \eta), \\
\Psi^{*}(\eta, k, I) & =\frac{1}{2} \Omega^{-1} \sqrt{\pi \eta} H_{\nu_{I}}^{(1)}(k \eta) .
\end{aligned}
$$

The propagators are obtained by substituting the mode functions above into the definition (35). In the coincidence limit $\left\|\vec{x}-\vec{x}^{\prime}\right\| \rightarrow 0, \eta-\eta^{\prime} \rightarrow 0$ we have, after performing a trivial angular integration,

$$
i \Delta_{I}(x)=\frac{1}{8 \pi} \frac{|\eta|}{\Omega^{2}} \int d k k^{2} e^{-\epsilon k} H_{\nu_{I}}^{(1)}(k \eta) H_{\nu_{I}}^{(2)}(k \eta) .
$$

Since $H_{\nu}^{(1,2)}(y) \propto y^{-1 / 2}$ when $y \rightarrow \infty$, the coincident propagators diverge as $k^{2}$ in the ultraviolet. After these divergences have been regularized at the initial value surface $\eta=\eta_{i}$, the counterterms should not affect the time evolution. Notice that the details of the regularization procedure and the ultraviolet behavior of the true, renormalizable quantum theory of gravity are issues immaterial to the long-range phenomena described by the effective theory, general relativity. 
We are mainly interested in the infrared behavior of the effective theory, since the physical mechanism behind back reaction is superadiabatic amplification of quantum fluctuations with physical wavelengths of the order of or bigger than the Hubble radius $H^{-1}$. We define the infrared as the scales larger than the Hubble scale,

$$
k_{p}=\frac{k}{\Omega}>H(\eta)=H_{\mathrm{i}} \Omega^{1-1 / s} .
$$

In the far infrared the Hankel functions $H_{\nu}^{(2)}$ can be approximated by

$$
H_{\nu}^{(2)}(k \eta)=\frac{\Gamma(\nu)}{i \pi}\left(\frac{2}{k \eta}\right)^{\nu}+\cdots,
$$

so at coincidence the infrared limit of the propagators (62) can be written as

$$
i \Delta_{I}^{(I R)}(x)=\frac{2^{2 \nu_{I}} \Gamma^{2}\left(\nu_{I}\right)}{8 \pi^{3}} \frac{1}{|\eta| \Omega^{2}} \int^{H_{\mathrm{i}} \Omega^{1-1 / s}} d k(k \eta)^{2-2 \nu_{I}} e^{-\varepsilon k} .
$$

One immediately sees that by Eqs. (55)-(57), for large $s$ the integral is infrared finite for the $B$ and $C$ modes, but it is divergent for the $A$ mode.

We can cure this infrared divergence by working on a compact spatial manifold. The momenta are then bounded from below, and the integrals above should be mode sums. If we set the size of the compact manifold to be the $H_{\mathrm{i}}$, the Hubble radius at the initial time $\eta_{i}$, the infrared cut-off is given by $k_{0}=H_{\mathrm{i}}$. In terms of physical wavelengths, the cut-off is the size of the initially inflating patch redshifted by the expansion of the universe, $\lambda_{0}^{\text {phys }}=2 \pi H_{i} \Omega$.

With the cut-off $k_{0}=H_{\mathrm{i}}$ bounding the integral (65) from below, the propagator $A$ is given in the infrared limit $(k>H \Omega)$ by

$$
i \Delta_{A}^{(I R)}(\eta)=\frac{H_{\mathrm{i}}^{2}}{8 \pi^{2}}(s-1)\left[\frac{2 \Gamma\left(\frac{3}{2}+\frac{1}{s-1}\right)}{\sqrt{\pi}}\left(\frac{s-1}{s}\right)^{\frac{s}{s-1}}\right]^{2}\left(1-\Omega^{-2 / s}\right)
$$

where the numerical factor between square brackets approaches 1 in the limit $s \rightarrow \infty$.

The propagators for the $B(+)$ and $C(-)$ modes in the infrared limit are best left as integrals, 


$$
i \Delta_{B, C}^{(I R)}=\frac{H_{\mathrm{i}}^{ \pm \frac{2}{s-1}}}{4 \pi^{2}}\left[\frac{\Gamma\left(\frac{1}{2} \pm \frac{1}{s-1}\right)}{\sqrt{\pi}}\left(\frac{s-1}{s}\right)^{ \pm \frac{1}{s-1}}\right]^{2} \Omega^{-2 \pm \frac{2}{s}} \int_{H_{\mathrm{i}}}^{H_{\mathrm{i}} \Omega^{1-1 / s}} d k k^{1 \mp \frac{2}{s-1}}
$$

where it is easy to see that they are dominated by the ultraviolet. In addition, they have an overall time factor of $\Omega^{-2 / s}$ which makes them subdominant when compared with the constant part of $i \Delta_{A}^{(I R)}$.

The dominant contribution from the infrared limit of the coincident propagators is therefore

$$
i \Delta_{A}^{(I R)}=\frac{H_{\mathrm{i}}^{2}}{8 \pi^{2}}(s-1)\left[\frac{2 \Gamma\left(\frac{3}{2}+\frac{1}{s-1}\right)}{\sqrt{\pi}}\left(\frac{s-1}{s}\right)^{\frac{s}{s-1}}\right]^{2} .
$$

\section{Results}

In this section we obtain and discuss the results for the expectation values of the metric and scalar field in the presence of the quantum fluctuations $\psi_{\mu \nu}$ and $\phi$.

Our strategy consists of summing up all contributions to the amputated 1-point functions (43) coming from cubic interactions, then obtaining the expectation values of the metric and scalar field by (47)-(49).

There are three types of vertices with cubic interactions in (19): pure graviton vertices $\psi^{3}$ (Table 1), graviton-ghost vertex $\psi \bar{w} w$ (Table 2) and vertices with one or more $\phi$ 's (Table 3 ).

For simplicity, we have partially symmetrized the $\psi^{3}$ vertices of Table 1 so that the first field always corresponds to the external line. For example, the following vertex

$$
\frac{1}{2} \kappa H_{\mathrm{i}} \Omega^{3-1 / s} \psi \psi_{, \alpha} \psi^{\alpha 0}
$$

gives rise to the symmetrized vertices numbers 1,2 or 3 of Table 1 when the first, third or second $\psi$ 's respectively are taken as the external leg.

As an example, consider the contributions to the amputated 1-point function $\alpha$ from the symmetrized vertex number 1. Using the Feynman rules of section 3 one obtains 


\begin{tabular}{|c|c|c|c|}
\hline$\#$ & Vertex Factor & $\#$ & Vertex Factor \\
\hline 1 & $\frac{1}{2} \kappa H_{\mathrm{i}} \Omega^{3-1 / s} \eta^{\alpha_{1} \beta_{1}} \eta^{\alpha_{2} \beta_{2}} \partial_{2}^{\left(\alpha_{3}\right.} t^{\left.\beta_{3}\right)}$ & 22 & $\frac{1}{2} \kappa \Omega^{2} \eta^{\alpha_{2}\left(\alpha_{3}\right.} \eta^{\left.\beta_{3}\right) \beta_{2}} \partial_{3}^{\left(\alpha_{1}\right.} \partial_{1}^{\left.\beta_{1}\right)}$ \\
\hline 2 & $\frac{1}{2} \kappa H_{\mathrm{i}} \Omega^{3-1 / s} \eta^{\alpha_{2} \beta_{2}} \eta^{\alpha_{3} \beta_{3}} \partial_{3}^{\left(\alpha_{1}\right.} t^{\left.\beta_{1}\right)}$ & 23 & $\frac{1}{2} \kappa \Omega^{2} \eta^{\alpha_{3}\left(\alpha_{1}\right.} \eta^{\left.\beta_{1}\right) \beta_{3}} \partial_{1}^{\left(\alpha_{2}\right.} \partial_{2}^{\left.\beta_{2}\right)}$ \\
\hline 3 & $\frac{1}{2} \kappa H_{\mathrm{i}} \Omega^{3-1 / s} \eta^{\alpha_{3} \beta_{3}} \eta^{\alpha_{1} \beta_{1}} \partial_{1}^{\left(\alpha_{2}\right.} t^{\left.\beta_{2}\right)}$ & 24 & $\frac{1}{2} \kappa \Omega^{2} \partial_{2}^{\left(\alpha_{1}\right.} \eta^{\left.\beta_{1}\right)\left(\alpha_{3}\right.} \partial_{3}^{\left.\beta_{3}\right)} \eta^{\alpha_{2} \beta_{2}}$ \\
\hline 4 & $-\kappa H_{\mathrm{i}} \Omega^{3-1 / s} \eta^{\alpha_{1}\left(\alpha_{2}\right.} \eta^{\left.\beta_{2}\right) \beta_{1}} \partial_{2}^{\left(\alpha_{3}\right.} t^{\left.\beta_{3}\right)}$ & 25 & $\frac{1}{2} \kappa \Omega^{2} \partial_{3}^{\left(\alpha_{2}\right.} \eta^{\left.\beta_{2}\right)\left(\alpha_{1}\right.} \partial_{1}^{\left.\beta_{1}\right)} \eta^{\alpha_{3} \beta_{3}}$ \\
\hline 5 & $-\kappa H_{\mathrm{i}} \Omega^{3-1 / s} \eta^{\alpha_{2}\left(\alpha_{3}\right.} \eta^{\left.\beta_{3}\right) \beta_{2}} \partial_{3}^{\left(\alpha_{1}\right.} t^{\left.\beta_{1}\right)}$ & 26 & $\frac{1}{2} \kappa \Omega^{2} \partial_{1}^{\left(\alpha_{3}\right.} \eta^{\left.\beta_{3}\right)\left(\alpha_{2}\right.} \partial_{2}^{\left.\beta_{2}\right)} \eta^{\alpha_{1} \beta_{1}}$ \\
\hline 6 & $-\kappa H_{\mathrm{i}} \Omega^{3-1 / s} \eta^{\alpha_{3}\left(\alpha_{1}\right.} \eta^{\left.\beta_{1}\right) \beta_{3}} \partial_{1}^{\left(\alpha_{2}\right.} t^{\left.\beta_{2}\right)}$ & 27 & $\frac{1}{2} \kappa \Omega^{2} \partial_{2}^{\left(\alpha_{1}\right.} \eta^{\left.\beta_{1}\right)\left(\alpha_{2}\right.} \partial_{3}^{\left.\beta_{2}\right)} \eta^{\alpha_{3} \beta_{3}}$ \\
\hline 7 & $-\kappa H_{\mathrm{i}} \Omega^{3-1 / s} t^{\left(\alpha_{3}\right.} \eta^{\left.\beta_{3}\right)\left(\alpha_{1}\right.} \partial_{2}^{\left.\beta_{1}\right)} \eta^{\alpha_{2} \beta_{2}}$ & 28 & $\frac{1}{2} \kappa \Omega^{2} \partial_{3}^{\left(\alpha_{2}\right.} \eta^{\left.\beta_{2}\right)\left(\alpha_{3}\right.} \partial_{1}^{\left.\beta_{3}\right)} \eta^{\alpha_{1} \beta_{1}}$ \\
\hline 8 & $-\kappa H_{\mathrm{i}} \Omega^{3-1 / s} t^{\left(\alpha_{1}\right.} \eta^{\left.\beta_{1}\right)\left(\alpha_{2}\right.} \partial_{3}^{\left.\beta_{2}\right)} \eta^{\alpha_{3} \beta_{3}}$ & 29 & $\frac{1}{2} \kappa \Omega^{2} \partial_{1}^{\left(\alpha_{3}\right.} \eta^{\left.\beta_{3}\right)\left(\alpha_{1}\right.} \partial_{2}^{\left.\beta_{1}\right)} \eta^{\alpha_{2} \beta_{2}}$ \\
\hline 9 & $-\kappa H_{\mathrm{i}} \Omega^{3-1 / s} t^{\left(\alpha_{2}\right.} \eta^{\left.\beta_{2}\right)\left(\alpha_{3}\right.} \partial_{1}^{\left.\beta_{3}\right)} \eta^{\alpha_{1} \beta_{1}}$ & 30 & $\frac{1}{8} \kappa \Omega^{2} \eta^{\alpha_{1} \beta_{1}} \eta^{\alpha_{2} \beta_{2}} \eta^{\alpha_{3} \beta_{3}} \partial_{2} \cdot \partial_{3}$ \\
\hline 10 & $\frac{1}{4} \kappa \Omega^{2} \eta^{\alpha_{1} \beta_{1}} \partial_{3}^{\left(\alpha_{2}\right.} \eta^{\left.\beta_{2}\right)\left(\alpha_{3}\right.} \partial_{2}^{\left.\beta_{3}\right)}$ & 31 & $\frac{1}{4} \kappa \Omega^{2} \eta^{\alpha_{1} \beta_{1}} \eta^{\alpha_{2} \beta_{2}} \eta^{\alpha_{3} \beta_{3}} \partial_{3} \cdot \partial_{1}$ \\
\hline 11 & $\frac{1}{4} \kappa \Omega^{2} \eta^{\alpha_{2} \beta_{2}} \partial_{1}^{\left(\alpha_{3}\right.} \eta^{\left.\beta_{3}\right)\left(\alpha_{1}\right.} \partial_{3}^{\left.\beta_{1}\right)}$ & 32 & $-\frac{1}{2} \kappa \Omega^{2} \eta^{\alpha_{1}\left(\alpha_{2}\right.} \eta^{\left.\beta_{2}\right) \beta_{1}} \eta^{\alpha_{3} \beta_{3}} \partial_{2} \cdot \partial_{3}$ \\
\hline 12 & $\frac{1}{4} \kappa \Omega^{2} \eta^{\alpha_{3} \beta_{3}} \partial_{2}^{\left(\alpha_{1}\right.} \eta^{\left.\beta_{1}\right)\left(\alpha_{2}\right.} \partial_{1}^{\left.\beta_{2}\right)}$ & 33 & $-\frac{1}{2} \kappa \Omega^{2} \eta^{\alpha_{2}\left(\alpha_{3}\right.} \eta^{\left.\beta_{3}\right) \beta_{2}} \eta^{\alpha_{1} \beta_{1}} \partial_{3} \cdot \partial_{1}$ \\
\hline 13 & $-\kappa \Omega^{2} \partial_{3}^{\left(\alpha_{1}\right.} \eta^{\left.\beta_{1}\right)\left(\alpha_{2}\right.} \eta^{\left.\beta_{2}\right)\left(\alpha_{3}\right.} \partial_{2}^{\left.\beta_{3}\right)}$ & 34 & $-\frac{1}{2} \kappa \Omega^{2} \eta^{\alpha_{3}\left(\alpha_{1}\right.} \eta^{\left.\beta_{1}\right) \beta_{3}} \eta^{\alpha_{2} \beta_{2}} \partial_{1} \cdot \partial_{2}$ \\
\hline 14 & $-\kappa \Omega^{2} \partial_{1}^{\left(\alpha_{2}\right.} \eta^{\left.\beta_{2}\right)\left(\alpha_{3}\right.} \eta^{\left.\beta_{3}\right)\left(\alpha_{1}\right.} \partial_{3}^{\left.\beta_{1}\right)}$ & 35 & $-\frac{1}{4} \kappa \Omega^{2} \partial_{2}^{\left(\alpha_{1}\right.} \partial_{3}^{\left.\beta_{1}\right)} \eta^{\alpha_{2} \beta_{2}} \eta^{\alpha_{3} \beta_{3}}$ \\
\hline 15 & $-\kappa \Omega^{2} \partial_{2}^{\left(\alpha_{3}\right.} \eta^{\left.\beta_{3}\right)\left(\alpha_{1}\right.} \eta^{\left.\beta_{1}\right)\left(\alpha_{2}\right.} \partial_{1}^{\left.\beta_{2}\right)}$ & 36 & $-\frac{1}{2} \kappa \Omega^{2} \partial_{3}^{\left(\alpha_{2}\right.} \partial_{1}^{\left.\beta_{2}\right)} \eta^{\alpha_{3} \beta_{3}} \eta^{\alpha_{1} \beta_{1}}$ \\
\hline 16 & $-\frac{1}{2} \kappa \Omega^{2} \partial_{3}^{\left(\alpha_{2}\right.} \eta^{\left.\beta_{2}\right)\left(\alpha_{1}\right.} \eta^{\left.\beta_{1}\right)\left(\alpha_{3}\right.} \partial_{2}^{\left.\beta_{3}\right)}$ & 37 & $-\frac{1}{8} \kappa \Omega^{2} \eta^{\alpha_{1} \beta_{1}} \eta^{\alpha_{2}\left(\alpha_{3}\right.} \eta^{\left.\beta_{3}\right) \beta_{2}} \partial_{2} \cdot \partial_{3}$ \\
\hline 17 & $-\kappa \Omega^{2} \partial_{1}^{\left(\alpha_{3}\right.} \eta^{\left.\beta_{3}\right)\left(\alpha_{2}\right.} \eta^{\left.\beta_{2}\right)\left(\alpha_{1}\right.} \partial_{3}^{\left.\beta_{1}\right)}$ & 38 & $-\frac{1}{4} \kappa \Omega^{2} \eta^{\alpha_{2} \beta_{2}} \eta^{\alpha_{3}\left(\alpha_{1}\right.} \eta^{\left.\beta_{1}\right) \beta_{3}} \partial_{3} \cdot \partial_{1}$ \\
\hline 18 & $-\frac{1}{4} \kappa \Omega^{2} \eta^{\alpha_{1} \beta_{1}} \eta^{\alpha_{2} \beta_{2}} \partial_{2}^{\left(\alpha_{3}\right.} \partial_{3}^{\left.\beta_{3}\right)}$ & 39 & $\frac{1}{2} \kappa \Omega^{2} \eta^{\left.\alpha_{1}\right)\left(\alpha_{2}\right.} \eta^{\left.\beta_{2}\right)\left(\alpha_{3}\right.} \eta^{\left.\beta_{3}\right)\left(\beta_{1}\right.} \partial_{2} \cdot \partial_{3}$ \\
\hline 19 & $-\frac{1}{4} \kappa \Omega^{2} \eta^{\alpha_{2} \beta_{2}} \eta^{\alpha_{3} \beta_{3}} \partial_{3}^{\left(\alpha_{1}\right.} \partial_{1}^{\left.\beta_{1}\right)}$ & 40 & $\kappa \Omega^{2} \eta^{\left.\alpha_{1}\right)\left(\alpha_{2}\right.} \eta^{\left.\beta_{2}\right)\left(\alpha_{3}\right.} \eta^{\left.\beta_{3}\right)\left(\beta_{1}\right.} \partial_{3} \cdot \partial_{1}$ \\
\hline 20 & $-\frac{1}{4} \kappa \Omega^{2} \eta^{\alpha_{3} \beta_{3}} \eta^{\alpha_{1} \beta_{1}} \partial_{1}^{\left(\alpha_{2}\right.} \partial_{2}^{\left.\beta_{2}\right)}$ & 41 & $\frac{1}{4} \kappa \Omega^{2} \partial_{2}^{\left(\alpha_{1}\right.} \partial_{3}^{\left.\beta_{1}\right)} \eta^{\alpha_{2}\left(\alpha_{3}\right.} \eta^{\left.\beta_{3}\right) \beta_{2}}$ \\
\hline 21 & $\frac{1}{2} \kappa \Omega^{2} \eta^{\alpha_{1}\left(\alpha_{2}\right.} \eta^{\left.\beta_{2}\right) \beta_{1}} \partial_{2}^{\left(\alpha_{3}\right.} \partial_{3}^{\left.\beta_{3}\right)}$ & 42 & $\frac{1}{2} \kappa \Omega^{2} \partial_{3}^{\left(\alpha_{2}\right.} \partial_{1}^{\left.\beta_{2}\right)} \eta^{\alpha_{3}\left(\alpha_{1}\right.} \eta^{\left.\beta_{1}\right) \beta_{3}}$ \\
\hline
\end{tabular}

Table 1: Vertex factors contracted into $\psi_{\alpha_{1} \beta_{1}} \psi_{\alpha_{2} \beta_{2}} \psi_{\alpha_{3} \beta_{3}}$ with \#1 external. 


\begin{tabular}{|c|c|c|c|}
\hline$\#$ & Vertex Factor & $\#$ & Vertex Factor \\
\hline 1 & $-\kappa \Omega^{2} \eta^{\alpha_{2}\left(\alpha_{1}\right.} \eta^{\left.\beta_{1}\right) \alpha_{3}} \partial_{2} \cdot \partial_{3}$ & 6 & $\frac{1}{2} \kappa \Omega^{2} \eta^{\alpha_{1} \beta_{1}} \partial_{2}^{\alpha_{2}} \partial_{1}^{\alpha_{3}}$ \\
\hline 2 & $-\kappa \Omega^{2} \eta^{\alpha_{3}\left(\alpha_{1}\right.} \partial_{2}^{\left.\beta_{1}\right)} \partial_{3}^{\alpha_{2}}$ & 7 & $-\kappa H_{\mathrm{i}} \Omega^{3} \eta^{\alpha_{1} \beta_{1}} \partial_{2}^{\alpha_{2}} t^{\alpha_{3}}$ \\
\hline 3 & $-\kappa \Omega^{2} \eta^{\alpha_{2}\left(\alpha_{1}\right.} \partial_{2}^{\left.\beta_{1}\right)} \partial_{1}^{\alpha_{3}}$ & 8 & $-2 \kappa H_{\mathrm{i}} \Omega^{3} \eta^{\alpha_{3}\left(\alpha_{1}\right.} \partial_{3}^{\left.\beta_{1}\right)} t^{\alpha_{2}}$ \\
\hline 4 & $2 \kappa H_{\mathrm{i}} \Omega^{3} \eta^{\alpha_{2}\left(\alpha_{1}\right.} \partial_{2}^{\left.\beta_{1}\right)} t^{\alpha_{3}}$ & 9 & $-\kappa H_{\mathrm{i}} \Omega^{3} \eta^{\alpha_{1} \beta_{1}} \partial_{1}^{\alpha_{3}} t^{\alpha_{2}}$ \\
\hline 5 & $\kappa \Omega^{2} \eta^{\alpha_{3}\left(\alpha_{1}\right.} \partial_{3}^{\left.\beta_{1}\right)} \partial_{2}^{\alpha_{2}}$ & 10 & $2 \kappa H_{\mathrm{i}}^{2} \Omega^{4} \eta^{\alpha_{1} \beta_{1}} t^{\alpha_{2}} t^{\alpha_{3}}$ \\
\hline
\end{tabular}

Table 2: Vertex factors contracted into $\psi_{\alpha_{1} \beta_{1}} \bar{\omega}_{\alpha_{2}} \omega_{\alpha_{3}}$.

\begin{tabular}{|c|c|c|c|}
\hline$\#$ & Interaction & $\#$ & Interaction \\
\hline 1 & $\frac{1}{4 \sqrt{s}} \kappa H_{i} \Omega^{3-\frac{1}{s}} \phi^{\prime} \psi^{2}$ & 6 & $\frac{1}{2} \kappa \Omega^{2} \phi_{, \rho} \phi_{, \sigma} \psi^{\rho \sigma}$ \\
\hline 2 & $-\frac{1}{2 \sqrt{s}} \kappa H_{i} \Omega^{3-\frac{1}{s}} \phi^{\prime} \psi^{\rho \sigma} \psi_{\rho \sigma}$ & 7 & $\frac{1}{4 \sqrt{s}}\left(3-\frac{1}{s}\right) \kappa H_{i}^{2} \Omega^{4-\frac{2}{s}} \phi \psi^{2}$ \\
\hline 3 & $-\frac{1}{\sqrt{s}} \kappa H_{i} \Omega^{3-\frac{1}{s}} t_{\rho} \phi_{, \sigma} \psi^{\rho \sigma} \psi$ & 8 & $-\frac{1}{2 \sqrt{s}}\left(3-\frac{1}{s}\right) \kappa H_{i}^{2} \Omega^{4-\frac{2}{s}} \phi \psi^{\rho \sigma} \psi_{\rho \sigma}$ \\
\hline 4 & $\frac{2}{\sqrt{s}} \kappa H_{i} \Omega^{3-\frac{1}{s}} t_{\rho} \phi_{, \sigma} \psi^{\rho \mu} \psi_{\mu}^{\sigma}$ & 9 & $-\frac{1}{2 s}\left(3-\frac{1}{s}\right) \kappa H_{i}^{2} \Omega^{4-\frac{2}{s}} \phi^{2} \psi$ \\
\hline 5 & $-\frac{1}{4} \kappa \Omega^{2} \phi_{, \rho} \phi^{, \rho} \psi$ & 10 & $\frac{1}{2 s^{3 / 2}}\left(3-\frac{1}{s}\right) \kappa H_{i}^{2} \Omega^{4-\frac{2}{s}} \phi^{3}$ \\
\hline & & 11 & $\frac{2}{\sqrt{s}} \kappa H_{i} \Omega^{3-\frac{1}{s}} t_{\rho} \phi_{, \sigma} \bar{\omega}^{\rho} \omega^{\sigma}$ \\
\hline
\end{tabular}

Table 3: Cubic interactions involving $\phi$. 


$$
\begin{aligned}
\alpha_{[1]}(\eta) & =i \times i \kappa \times \frac{1}{2} \kappa H_{\mathrm{i}} \Omega^{3-1 / s}\left[\partial^{\alpha}(x)\left\langle\psi(x) \psi_{0 \alpha}\left(x^{\prime}\right)\right\rangle\right]_{x^{\prime} \rightarrow x} \\
& =-\kappa^{2} H_{\mathrm{i}} \Omega^{3-1 / s} \partial_{\eta}\left[-\frac{2}{s+1} i \Delta_{A}\left(x ; x^{\prime}\right)-\frac{2 s}{s+1} i \Delta_{C}\left(x ; x^{\prime}\right)\right]_{x^{\prime} \rightarrow x}
\end{aligned}
$$

We are looking for those terms that grow the fastest as a function of time, such that after integration by (52) or (53) there can be a sustained effect of back reactions on the metric and scalar field $A, C$ and $D$. Clearly, the vertex above fails to meet that condition and contributes at maximum a constant to the expectation values.

The terms which we are interested in arise when a time derivative hits an external leg, as happens in vertex number 3, for example. When that term is integrated by parts and the conformal time derivative hits the volume factor $H_{\mathrm{i}} \Omega^{3-1 / s}$, the result is a factor of $H_{\mathrm{i}}^{2} \Omega^{4-2 / s}$. This sort of term, when integrated with respect to the inverse of the $A$-type propagator by (52), gives a dominant logarithmic contribution.

On the other hand, when vertex number 3 is integrated with respect to the inverse of the $C$-type propagator by (53), the result is only a constant and other subdominant terms. Since integration by the $C$-type propagator gives only subdominant contributions, we ignore them in what follows.

It can be verified by inspection that no terms with factors that grow faster than $\Omega^{4-2 / s}$ arise in the Einstein Lagrangian. Those are the types of contributions we are looking for, and only the terms with the right structure to contribute a factor of at least $H_{\mathrm{i}}^{2} \Omega^{4-2 / s}$ will be collected in the following expressions.

Our results for the amputated 1-point functions to leading order are as follows:

$$
\begin{aligned}
\alpha(\eta) & =-\frac{1}{2} \frac{1}{s+1}\left(3-\frac{1}{s}\right) \kappa^{2} H_{\mathrm{i}}^{2} \Omega^{4-2 / s} i \Delta_{A}^{(I R)} \\
\gamma(\eta) & =-\alpha(\eta) \\
\delta(\eta) & =-\frac{2}{\sqrt{s}} \alpha(\eta)
\end{aligned}
$$

where $i \Delta_{A}^{(I R)}$ is given in expression (68). 
The expectation values can be found with the help of expressions (47)(49) and (52):

$$
\begin{aligned}
A(\eta)= & -\frac{\kappa^{2} H_{\mathrm{i}}^{2}}{8 \pi^{2}} \frac{s^{2}-s}{(s+1)^{2}}\left[\frac{2 \Gamma\left(\frac{3}{2}+\frac{1}{s-1}\right)}{\sqrt{\pi}}\left(\frac{s-1}{s}\right)^{\frac{s}{s-1}}\right]^{2} \\
& \times\left[\ln \Omega-\frac{s}{3 s-1}+\frac{s}{3 s-1} \Omega^{-\frac{3 s-1}{s}}\right] \\
C(\eta)= & -\frac{1}{s} A(\eta) \\
\frac{D(\eta)}{\sqrt{s}}= & \frac{1}{s} A(\eta)
\end{aligned}
$$

where we keep the subdominant terms after the logarithm only to stress that expectation values vanish at the initial value surface $\Omega=1$. The prefactor in square brackets of expression (75), which we call $\sigma(s)$, is approximately 1 for large $s$.

The effective Hubble expansion parameter can be evaluated by substituting the expressions above for the expectation value of the metric into Eq. (42). It can be easily seen that the logarithmic contributions cancel, and all that remains are subdominant terms. The effective expansion rate is unchanged up to terms which are either constant and decaying as functions of time,

$$
H_{\text {eff }}(\tilde{t})=H(t)[1+(\text { subdominant })] .
$$

We conclude therefore that there is no one loop back reaction of quantum fluctuations on power law inflation to leading order. Presumably there is a two loop effect, analogous to the one found by Tsamis and Woodard [6], that grows and would become dominant, but we did not attempt to calculate those diagrams.

In the next section we will check the results above with a vastly simpler canonical version of this calculation, and provide a physical justification for the differences among the power-law and chaotic inflation cases. 


\section{The canonical result}

As a check on our previous results, and in its own right, it is interesting to study the same problem of back reaction in power-law inflation using canonical quantization. This was the method used by Mukhanov et al. to derive their results.

In order to simplify the full Lagrangian (11), we perform the following approximations: first, ignore the spin-2 (gravity waves) and spin-1 ("vector" fluctuations) projections of the graviton degrees of freedom, and concentrate on the scalar fluctuations of the metric that couple to the fluctuations of the scalar field $\varphi$. Second, use the constraints of the Einstein field equations explicitly in (1) to eliminate the fluctuations of the scalar field and reduce the number of degrees of freedom to one: the so-called Newtonian potential $\Phi$. Third, the expectation values are given in terms of the "spectrum" of the canonically quantized field $\Phi$ in power-law inflation, which can be read right off the standard formulas in the literature on quantum fluctuations in inflationary universe models [9, 12].

The motivation for the first assumption lies in the observation that the infrared limits of coincident propagators of the spin- 1 and spin- 2 degrees of freedom are dominated by the ultraviolet and fall off as a function of time. Since cross-correlations are irrelevant at one loop, we can ignore those degrees of freedom altogether and concentrate on the dominant, scalar degrees of freedom of the graviton.

This truncated version of canonical quantization has obvious shortcomings, such as its inadequacy to study perturbative corrections above lowest order and the exclusion of many of the degrees of freedom from the calculation. These difficulties would be unsurmountable if we wanted to to calculate quantum corrections beyond one-loop, for example. Nevertheless, within the scope of this one loop calculation the truncated canonical method is perfectly suited to study the leading contributions to back reaction.

The back reaction on the homogeneous and isotropic background is determined by the expectation values of Einstein's field equations

$$
\left\langle 0\left|G_{\nu}^{\mu}\right| 0\right\rangle=\frac{\kappa^{2}}{2}\left\langle 0\left|T_{\nu}^{\mu}\right| 0\right\rangle
$$

and the equation of motion for the scalar field,

$$
\left\langle 0\left|\square \varphi+V_{, \varphi}\right| 0\right\rangle=0
$$


where the inflaton potential is

$$
V(\varphi)=6\left(1-\frac{1}{3 s}\right) \frac{H_{\mathrm{i}}^{2}}{\kappa^{2}} \exp \left[-\frac{\kappa}{\sqrt{s}} \varphi\right] .
$$

The terms in Einstein's equations which are quadratic in the quantum fluctuations can be collected in an effective stress-energy tensor $\tau_{\mu \nu}$, which is a source term in addition to the stress-energy tensor of the background matter [3, 4]. The equation of motion for $\varphi$ is similarly corrected by source terms quadratic in the quantum fields. In this section we shall regard back reaction as the response of the background to the source terms induced by quantum fluctuations, found by solving for the expectation values of Einstein's equations and the equation of motion for the scalar field.

We write the metric in longitudinal gauge,

$$
d s^{2}=-[1+2 \Phi(t, \vec{x})] d t^{2}+a_{0}^{2}(t)[1-2 \Phi(t, \vec{x})+2 w(t)] d \vec{x} \cdot d \vec{x}
$$

and the scalar field is

$$
\varphi=\varphi_{0}(t)+\phi(t, \vec{x})+v(t),
$$

where $\Phi$ and $\phi$ are, as before, quantum fields which ought to be canonically quantized, and $w(t)$ and $v(t)$ are respectively corrections to the scale factor and to the scalar field from back reaction?2 We have taken advantage of the freedom of gauge at the second order to fix any correction to the time slicing (the $N$ of earlier sections) to zero. Therefore, in this gauge $\dot{w}$ is the correction to the Hubble expansion rate, $H_{\mathrm{eff}}=H+\dot{w}$ that will eventually be compared with the effective expansion rate $(\overline{78})$ of the last section.

The spectrum $\left|\delta_{\Phi}(k, t)\right|$ is defined by the expectation value of the square of the canonically quantized Newtonian potential:

$$
\langle 0|\Phi(t, \vec{x}) \Phi(t, \vec{x})| 0\rangle=\int \frac{d k}{k}\left|\delta_{\Phi}(k, t)\right|^{2},
$$

The spectrum is proportional to $k$ when $k \rightarrow \infty$, and consequently the expectation value above diverges as $k^{2}$ in the far ultraviolet. Just as was

\footnotetext{
${ }^{2}$ We have adopted a notation different from earlier sections to avoid confusion, since we in the section we work in a different gauge
} 
done in section 5, we assume that a proper renormalization of these infinities have been performed and that the appropriate counterterms have no bearing on the long-range interactions described by the effective theory.

The expectation value (84) is also divergent in the infrared since the spectrum is approximately constant for very small momenta. Again the solution is to work in a compact spatial manifold, where $k$ is cut off at the value $H_{\mathrm{i}}$ corresponding to the radius of the manifold at the initial time $t=0$.

The infrared limit of the spectrum of the Newtonian potential during power-law inflation is 12

$$
\left|\delta_{\Phi}^{(I R)}(k, t)\right|^{2}=\frac{\kappa^{2} H_{\mathrm{i}}^{2}}{32 \pi^{2}} \frac{\sigma(s)}{s}\left(\frac{k}{H_{\mathrm{i}}}\right)^{-\frac{2}{s-1}}
$$

where

$$
\sigma(s)=\left[\frac{2 \Gamma\left(\frac{3}{2}+\frac{1}{s-1}\right)}{\sqrt{\pi}}\left(\frac{s-1}{s}\right)^{\frac{s}{s-1}}\right]^{2},
$$

is the same factor defined below (75), and approaches 1 as $s \gg 1$.

The physical information contained in this spectrum is that the amplitudes of long-wavelength fluctuations are asymptotically constant in powerlaw inflation. The amplitudes of infrared fluctuations in chaotic inflation, by comparison, grow slowly with time. Where back reaction is concerned, quantum corrections to power-law inflation from a fluctuation with a fixed comoving wavelength can be at most constant, while corrections to the background in chaotic models can grow as a function of time during the inflation of the universe.

The distinguishing facts about power-law inflation are 1) the equation of state $w \equiv \rho / p=-1+2 / 3 s$ is constant throughout inflation, and 2) the kinetic and potential energy densities of the background scalar field, as well as the Hubble parameter, are at fixed ratios with respect to each other at any given time. Consequently, the amplitude of fluctuations on large scales, which couple to these ratios, freeze to a constant value.

We often find claims in the literature to the effect that quantum fluctuations during inflation freeze after they become larger than the Hubble radius. While this is exact for power-law inflation, it is only approximate for most models of inflation. 
With the spectra above, the expectation value of the Newtonian potential in the infrared limit is, by (84),

$$
\langle 0|\Phi(t, \vec{x}) \Phi(t, \vec{x})| 0\rangle=\sigma(s) \frac{s-1}{s} \frac{\kappa^{2} H_{\mathrm{i}}^{2}}{32 \pi^{2}}=\text { const } .
$$

Notice that this is in accord with expressions (36) and (68) for large $s$ (remember that $\left.\psi_{00}=2 \Phi\right)$. The expectation value of the scalar field can be deduced from this expression by using the following useful constraint in momentum space, valid in longitudinal gauge:

$$
\phi(t, k)=-2 \frac{V}{V_{, \varphi}} \Phi(t, k)=2 \frac{\sqrt{s}}{\kappa} \Phi(t, k) .
$$

The Einstein equations with quantum corrections are found by using the metric (82) and scalar field (83) into Eqs. (79). The general result (the spatial gradient terms have been ignored since we consider only infrared modes) is:

$$
\begin{aligned}
3 H^{2}+ & 3\left\langle\dot{\Phi}^{2}\right\rangle+12 H^{2}\left\langle\Phi^{2}\right\rangle+6 H \dot{w}=\frac{\kappa^{2}}{2}\left[\frac{1}{2} \dot{\varphi}_{0}^{2}+V\right. \\
+ & \left.\frac{1}{2}\left(\left\langle\dot{\phi}^{2}\right\rangle-4 \dot{\varphi}_{0}\langle\dot{\phi} \Phi\rangle+4 \dot{\varphi}_{0}^{2}\left\langle\Phi^{2}\right\rangle+2 \dot{\varphi}_{0} \dot{v}\right)+\frac{1}{2} V_{, \varphi \varphi}\left\langle\phi^{2}\right\rangle+V_{, \varphi} v\right], \\
3 H^{2}+ & 2 \dot{H}+4\left(H^{2}+2 \dot{H}\right)\left\langle\Phi^{2}\right\rangle+8 H\langle\dot{\Phi} \Phi\rangle+\left\langle\dot{\Phi}^{2}\right\rangle+6 H \dot{w}+2 \ddot{w} \\
= & \frac{\kappa^{2}}{2}\left[-\frac{1}{2} \dot{\varphi}_{0}^{2}+V-\frac{1}{2}\left(\left\langle\dot{\phi}^{2}\right\rangle-4 \dot{\varphi}_{0}\langle\dot{\phi} \Phi\rangle+4 \dot{\varphi}_{0}^{2}\left\langle\Phi^{2}\right\rangle+2 \dot{\varphi}_{0} \dot{v}\right)\right. \\
& \left.+\frac{1}{2} V_{, \varphi \varphi}\left\langle\phi^{2}\right\rangle+V_{, \varphi} v\right]
\end{aligned}
$$

where brackets $\langle\cdots\rangle$ denote vacuum expectation values.

As we have argued in the discussion of the spectrum, terms containing time derivatives of $\Phi$ and $\phi$ should vanish. Using the identity (88), we can finally write the Einstein equations with quantum corrections above in terms of only $\left\langle\Phi^{2}\right\rangle, w(t)$ and $v(t)$ :

$$
6 H \dot{w}=\frac{\kappa^{2}}{2}\left[\dot{\varphi}_{0} \dot{v}-V\left(\frac{\kappa v}{\sqrt{s}}+2\left\langle\Phi^{2}\right\rangle\right)\right],
$$




$$
6 H \dot{w}+2 \ddot{w}=\frac{\kappa^{2}}{2}\left[\dot{\varphi}_{0} \dot{v}-V\left(\frac{\kappa v}{\sqrt{s}}+2\left\langle\Phi^{2}\right\rangle\right)\right] .
$$

It is also instructive to write down the equation of motion for the scalar field to second order,

$$
\begin{aligned}
\ddot{v} & +3 H \dot{v}+3 \dot{w} \dot{\varphi}_{0}+V_{, \varphi \varphi} v \\
& -4\langle\dot{\Phi} \dot{\phi}\rangle-4 \dot{\varphi}_{0}\langle\dot{\Phi} \Phi\rangle+2 V_{, \varphi \varphi}\langle\Phi \phi\rangle+\frac{1}{2} V_{, \varphi \varphi \varphi}\left\langle\phi^{2}\right\rangle=0,
\end{aligned}
$$

which, after use of (88), the background identities and the Einstein field equation (91) read

$$
t^{2} \ddot{x}+\frac{3 s+1}{s} t \dot{x}+3 s\left(x+2\left\langle\Phi^{2}\right\rangle\right)=0,
$$

where $x=\kappa v / \sqrt{s}$. Since this equation is second order in time, we can always find solutions such that $v\left(t_{0}\right)=\dot{v}\left(t_{0}\right)=0$. Notice that $\left\langle\Phi^{2}\right\rangle$ is a constant by (87). It is straightforward to solve this equation, and the result is

$$
x=\frac{\kappa v}{\sqrt{s}}=-2\left[1-\frac{3 s-1}{3 s-2} \frac{t_{0}}{t}+\frac{1}{3 s-2}\left(\frac{t_{0}}{t}\right)^{3 s-1}\right]\left\langle\Phi^{2}\right\rangle,
$$

where we have chosen the integration constants such that at the initial value surface $x(0)=\dot{x}(0)=0$.

The dominant contribution to $x=\kappa v / \sqrt{s}$ is therefore a constant, corresponding to the homogeneous solution to (94). However, as happened in the covariant calculation, we have neglected subdominant terms which decay as a function of time in the present calculation, by discarding several of the degrees of freedom of the fundamental Lagrangian. Therefore, the inhomogeneous solutions are unreliable within our approximation scheme and will be discarded.

Substituting the homogeneous part of (95) into (92) we get the following equation for the correction to the scale factor:

$$
t^{2} \ddot{w}+3 s t \dot{w}=0 .
$$

The solution is also straightforward, and we get 


$$
\dot{w}=0
$$

exactly since the decaying solution $\dot{w} \propto t^{-3 s}$ has to be zero to satisfy the retarded boundary conditions.

The effective expansion rate is therefore given by,

$$
H_{\mathrm{eff}}=H(t)
$$

We have thus obtained the back reaction on the metric and scalar field by finding the solutions to the expectation values of the Einstein field equations (79) and the equation of motion for the scalar field (80). Expectation values were evaluated using the canonically quantized Newtonian potential $\Phi$.

The result of the canonical calculation is that the back reaction of quantum fluctuations during power-law inflation does not affect the expansion rate of the universe, at least to leading order. This is in agreement with the results of the covariant calculation of last section.

As pointed out earlier in this section, infrared fluctuations of a fixed comoving wavelength in power-law inflation have constant amplitudes. Those fluctuations exiting the Hubble radius at a later time in the inflation epoch have a smaller amplitude than the ones that exited earlier, since the scale of inflation is decreasing as $\propto t^{-2}$. The cumulative effect of superimposing modes of different comoving momenta is not sufficient to make the expectation values of the quantum fields grow in time, and the momentum mode sum is dominated by modes that exited the horizon early in the inflation epoch.

\section{Discussion}

We have calculated the back reaction of quantum fluctuations on the expansion rate of homogeneous backgrounds of power-law inflation models. Two methods were employed: covariant quantization of the full scalar-graviton system, and canonical quantization of a reduced system where the spin-1 and spin-2 degrees of freedom of the graviton were purged.

The results of the two calculations are identical: to leading order, there is no effect of the quantum fluctuations on the effective Hubble parameter in 
power-law inflation. The physical reason is that long wavelength modes have constant amplitudes, and therefore both the amputated 1-point functions and the source terms in the expectation values of Einstein's equations are constant.

The main result is that the shape of the inflaton potential can have an enormous impact on back reaction. While chaotic inflation can have a significant back reaction, power-law inflation does not.

We also hope that the results presented here (see also [8]) will help settle some of the criticisms raised with respect to the canonical method as applied to the study of back reaction in quantum general relativity.

\section{Acknowledgments}

It is a pleasure to acknowledge stimulating and informative conversations with R. H. Brandenberger and V. F. Mukhanov. We are also grateful to the University of Crete for its hospitality at the inception of this project. This work was partially supported by DOE contract DE-FG02-97ER41029, by NSF grant 94092715 , by NATO grant CRG-971166 and by the Institute for Fundamental Theory.

\section{References}

[1] J. Schwinger, J. Math. Phys. 2 (1961) 407; Particles, Sources and Fields (Addison-Wesley, Reading, MA, 1970).

[2] J. Iliopoulos, T. N. Tomaras, N. C. Tsamis and R. P. Woodard, Nucl. Phys. B534 (1998) 419.

[3] V. Mukhanov, L. R. W. Abramo and R. Brandenberger, Phys. Rev. Lett. 78 (1997) 1624.

[4] L. R. W. Abramo, R. H. Brandenberger and V. F. Mukhanov, Phys. Rev. D56 (1997) 3248.

[5] L. F. Abbott and M. B. Wise, Nucl. Phys. B244 (1984) 541.

[6] N. C. Tsamis and R. P. Woodard, Nucl. Phys. B474 (1996) 235; Ann. Phys. 253 (1997) 1. 
[7] W. Unruh, "Cosmological Long Wavelength Perturbations," astroph/9802323.

[8] L. R. Abramo and R. P. Woodard, "One Loop Back Reaction On Chaotic Inflation," astro-ph/9811430.

[9] V. Mukhanov, H. Feldman and R. Brandenberger, Phys. Rep. 215 (1992) 203.

[10] G. Feinberg and J. Sucher, Phys. Rev. 166 (1968) 1638.

[11] N. C. Tsamis and R. P. Woodard, Ann. Phys. 253 (1997) 1.

[12] D. H. Lyth and E. D. Stewart, Phys. Lett. B 274 (1992) 168.

[13] A. D. Linde, Phys. Lett. 129B (1983) 177. 